Article

\title{
Blockade of Lactate Dehydrogenase-A (LDH-A) Improves Efficacy of Anti-Programmed Cell Death-1 (PD-1) Therapy in Melanoma
}

\author{
Saeed Daneshmandi ${ }^{1,2}$, Barbara Wegiel ${ }^{3,4, *(D)}$ and Pankaj Seth ${ }^{1,2,4, *}$
}

1 Department of Medicine, Beth Israel Deaconess Medical Center, Harvard Medical School, Boston, MA 02215, USA; sdaneshm@bidmc.harvard.edu

2 Division of Interdisciplinary Medicine, Beth Israel Deaconess Medical Center, Boston, MA 02215, USA

3 Department of Surgery, Beth Israel Deaconess Medical Center, Harvard Medical School, Boston, MA 02215, USA

4 Cancer Research Institute, Beth Israel Deaconess Medical Center, Harvard Medical School, Boston, MA 02215, USA

* Correspondence: bwegiel@bidmc.harvard.edu (B.W.); pseth@bidmc.harvard.edu (P.S.); Tel.: +617-735-2846 (B.W.) +617-667-2029 (P.S.)

Received: 6 February 2019; Accepted: 23 March 2019; Published: 29 March 2019

\begin{abstract}
Immunotherapy is a curable treatment for certain cancers, but it is still only effective in a small subset of patients. We have recently reported that programmed cell death protein-1 (PD-1) ligand (PD-L1) expression is regulated by lactate present at high levels in the tumor microenvironment (TME). We hypothesized that the efficacy of anti-PD-1 treatment can be improved by blocking the lactate-generating enzyme, lactate dehydrogenase-A (LDH-A). Anti-PD-1 treatment of mice harboring LDH-A deficient B16-F10 melanoma tumors led to an increase in anti-tumor immune responses compared to mice implanted with tumors expressing LDH-A. Specifically, we observed heightened infiltration of natural killer (NK) cells and $\mathrm{CD} 8^{+}$cytotoxic $\mathrm{T}$ cells in the LDH-A deficient tumors. These infiltrated cytotoxic cells had an elevated production of interferon- $\gamma($ IFN- $\gamma$ ) and granzyme B. Mechanistically, CD8 ${ }^{+} \mathrm{T}$ cells isolated from the TME of LDH-A deficient B16-F10 melanoma tumors and treated with anti-PD-1 showed enhanced mitochondrial activity and increased reactive oxygen species (ROS) levels. Moreover, infiltration of T regulatory (Treg) cells was diminished in LDH-A deficient tumors treated with anti-PD-1. These altered immune cell profiles were clinically relevant as they were accompanied by significantly reduced tumor growth. Our study suggests that blocking LDH-A in the tumor might improve the efficacy of anti-PD-1 therapy.
\end{abstract}

Keywords: LDH-A; lactate; PD-1; PD-L1; melanoma

\section{Introduction}

Cancer cells rely on fermentative glycolysis to maintain their growth even under normoxic conditions, known as the Warburg effect [1]. Lactate dehydrogenase-A (LDH-A) is a key enzyme that catalyzes the last reaction of fermentative glycolysis, which converts pyruvate to lactate. LDH-A serves as a critical checkpoint within this pathway to regenerate nicotinamide adenine dinucleotide serves as a critical checkpoint within this pathway to regenerate nicotinamide adenine dinucleotide $\left(\mathrm{NAD}^{+}\right)$. LDH-A levels are elevated in several cancer types and correlate with cancer growth, metastasis and recurrence as well as a poor clinical outcome [2,3]. We have recently demonstrated the role of LDH-A in myeloid cells in the tumor microenvironment (TME) as a driver of carcinogenesis [4]. Lactate, a metabolic product of LDH-A, is considered as an oncometabolite and elevated levels of lactate in cancer are associated with an increased number of metastases and lower survival. Recent studies indicate that 
lactate and/or low intra-tumoral $\mathrm{pH}$ promote an immunosuppressive TME with reduced infiltration of natural killer (NK) cells and cytotoxic CD8 ${ }^{+} \mathrm{T}$ lymphocytes and suppression of interferon- $\gamma$ (IFN- $\gamma$ ) expression [5]. In vitro exposure to lactate and/or acidification of the culture medium suppress proliferation and cytokine production in human cytotoxic T lymphocytes (CTLs) and can be restored by increasing the $\mathrm{pH}$ [6]. Moreover, raising intra-tumoral $\mathrm{pH}$ with oral bicarbonate treatment can improve the anti-tumoral effect of immunotherapy in preclinical settings [7]. Together, these observations strongly suggest that decreasing lactate production in cancer cells, thereby preventing acidification of the TME, might synergize with immunotherapy. Recently, we reported that LDH-A-derived lactate within the TME upregulates expression of programmed cell death protein-1 (PD-1) ligand (PD-L1) on tumor cells [4]. The PD-1/PD-L1 pathway is one of the dominant immune checkpoints in the TME. Tumor cells express PD-L1, which suppresses T cell proliferation and effector function by ligating PD-1 expressed on activated T cells [8]. Beneficial effects of PD-1/PD-L1 blockade have been reported in several cancer types including breast cancer, melanoma, non-small cell lung, Hodgkin's lymphoma, bladder, and kidney cancer [9]. However, the high efficacy of this treatment is limited to a subset of patients.

In this study, we investigated the role of LDH-A expressed in tumors for modulating immune responses to anti-PD-1 therapy in a B16-F10 melanoma model. We hypothesized that PD-1 blockade using an anti-PD-1 antibody might further inhibit the effects of the remaining PD-L1 expressed on tumor cells and block PD-1 signaling on immune cells. We demonstrate that deletion of LDH-A in melanoma cells abrogated PD-L1 expression and resulted in increased intra-tumoral immune cell infiltrates. These effects were assessed in the TME and were significantly enhanced by PD-1 blockade resulting in reduced tumor growth. These results also were confirmed by in vitro co-culture of $\mathrm{CD} 8^{+}$ $\mathrm{T}$ cells with melanoma cells, resulting in enhancement of degranulation markers and mitochondrial function in the cultures of melanoma cells with the deletion of LDH-A with T cells.

\section{Results}

\subsection{Treatment of Mice Harboring LDH-A Deficient B16-F10 Tumors with Anti-PD-1 Shows Reduced Tumor Growth, Lower Angiogenesis and Enhanced T Cell Infiltration}

We have previously reported that exogenous lactate increases PD-L1 expression in Lewis lung carcinoma (LLC) [4]. We demonstrate the same effect of exogenous lactate on melanoma cells (Figure 1A). We generated LDH-A deficient B16-F10 tumor cells (shLDH-A) by targeting LDH-A transcript with small hairpin RNA (shRNA). Control scramble shRNA was used to generate the appropriate control melanoma cell line. Low LDH-A protein levels in shLDH-A melanoma cells compared to control cells were confirmed by Western blot (Figure 1B and Supplementary Figure S1A). Similarly, diminished capacity for lactate production was confirmed in shLDH-A melanoma cells by colorimetric assay (Figure 1C). shLDH-A melanoma cells had significantly decreased PD-L1 expression compared to control cells (Figure 1D). Addition of exogenous lactate could override the effect of LDH-A deletion on the decrease of PD-L1 expression (Figure 1D) providing evidence for a direct causative link between lactate production and PD-L1 expression level.

To determine the in vivo biological implications of our findings, we examined whether the shLDH-A B16-F10 melanoma cells had a different capacity to induce tumors in vivo compared to the control B16-F10 melanoma cells. shLDH-A and control B16-F10 melanoma cells were injected subcutaneously into syngeneic C57BL/6 mice and each group was divided into two subsets, which were treated either with the anti-PD-1 antibody or an isotype control. We confirmed the deletion of LDH-A in the tumors (Supplementary Figure S1B). Anti-PD-1 antibody treatment in recipients of control B16-F10 melanoma cells reduced tumor growth, consistent with previous observations [10]. Deletion of LDH-A in B16-F10 melanoma cells reduced tumor growth and treatment with anti-PD-1 antibody almost completely abrogated tumor growth in this group of mice (Figure $1 \mathrm{~F}-\mathrm{G}$ ), suggesting an additive effect of both manipulations. Our immunostaining shows that shLDH-A deficient tumors were characterized by lower PD-L1 staining and had decreased proliferation and angiogenesis (Supplementary Figure S2). 

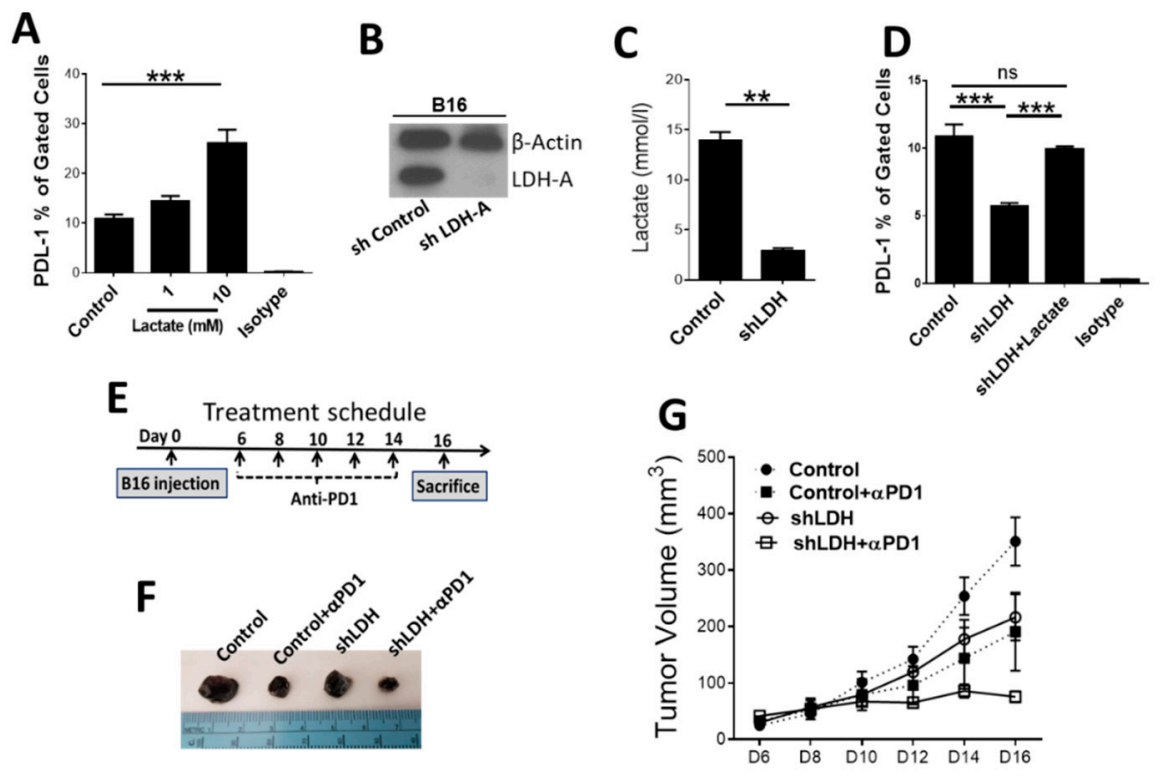

Figure 1. Lactate induces expression of programmed cell death protein-1 ligand (PD-L1) on B16 melanoma cells and deletion of lactate dehydrogenase-A (LDH-A) in B16 cells or anti-PD-1 therapy prevents tumor growth. (A) B16-F10 cells were treated with lactate (1 mM and $10 \mathrm{mM})$ for $24 \mathrm{~h}$, and the level of PD-L1 was measured by flow cytometry. The number of PD-L1 ${ }^{+}$cells is shown as the percentage of gated cells. ${ }^{* * *} p<0.001$. (B) LDH-A was knocked down in B16-F10 melanoma cells using small hairpin RNA (shRNA) against LDH-A. Scramble shRNA was used as control. Western blotting with an antibody against LDH-A is shown on B16-green fluorescent protein (GFP) versus shLDH melanoma cells. (C) Capacity of lactate production was examined on B16-shLDH and control melanoma cells. ${ }^{* *} p<0.01$. (D) B16-GFP and B16-shLDH melanoma cells were cultured in vitro and PD-L1 expression in the cells was measured by flow cytometry. ${ }^{* * *} p<0.001, n=3$ independent experiments in triplicate. (E) Anti-PD-1 treatment schedule of mice tumor model. (F-G) Anti-PD-1 administration provided the same tumor growth inhibition as B16-shLDH tumor growth and anti-PD-1 injection induced additional tumor growth inhibition $(p<0.05)$. (F) demonstrates representative tumor size at day 16 after induction of tumor. ns: not significant.

\subsection{Anti-PD-1 Therapy Induces Cytotoxic Responses in the TME of LDH-A Deficient B16-F10 Tumors}

Inhibition of tumor growth by blockade of the PD-1/PD-L1 pathway correlates with the increase of $\mathrm{T}$ cell subsets in the TME [11]. To examine whether the observed difference in tumor growth in vivo was associated with distinct cell infiltrates, we examined fractions of $\mathrm{CD}^{+}$and $\mathrm{CD} 8^{+} \mathrm{T}$ cells, NK cells and T regularity (Treg) cells in shLDH-A deficient and control B16-F10 tumors (Figure 2). Fractions of $\mathrm{CD}^{+}$and $\mathrm{CD}^{+}$tumor infiltrating $\mathrm{T}$ cells were significantly increased after PD-1 antibody treatment only in recipients of shLDH-A deficient B16-F10 tumors. Notably, no significant increase in CD4 ${ }^{+}$and $\mathrm{CD}^{+}$tumor infiltrating $\mathrm{T}$ cells was observed between the PD-1 Ab-treated and isotype control-treated mice bearing LDH-A expressing B16-F10 tumors (Figure 2A,B). We showed a similar pattern using immunostaining with anti-CD8 antibodies in the TME (Supplementary Figure S3). We also observed similar pattern of changes in $\mathrm{NK}\left(\mathrm{NK} 1.1^{+}\right)$tumor infiltrating $\mathrm{T}$ cells in mice bearing shLDH-A and control B16-F10 tumors, treated with a PD-1 blocking (Figure 2C). The number of NK (NK1.1 $)$ tumor infiltrating $\mathrm{T}$ cells was also increased in mice bearing shLDH-A B16-F10 tumors compared to those bearing control B16-F10 tumors even without treatment with the PD-1 blocking antibody (Figure 2C). This finding reveals the direct effect of lactate on the migration or expansion of NK T cells in the TME.

An inverse profile was observed in the infiltrates of Treg cells in the TME, which were significantly decreased in the context of shLDH-A B16-F10 compared to control B16-F10 tumors even without anti-PD-1 antibody treatment. A lower number of Treg cells in this group was further enhanced by anti-PD-1 antibody treatment (Figure 2D). The calculation of the $\mathrm{CD} 8^{+} /$Treg ratio demonstrates a higher 
trend in combination of LDH-A blocking and anti-PD-1 application (Figure 2E). We also detected an increased population of interleukin (IL)-17A ${ }^{+} \mathrm{CD}^{+}$(TC17) cells in the spleens of mice after anti-PD-1 treatment (Supplementary Figure S4).
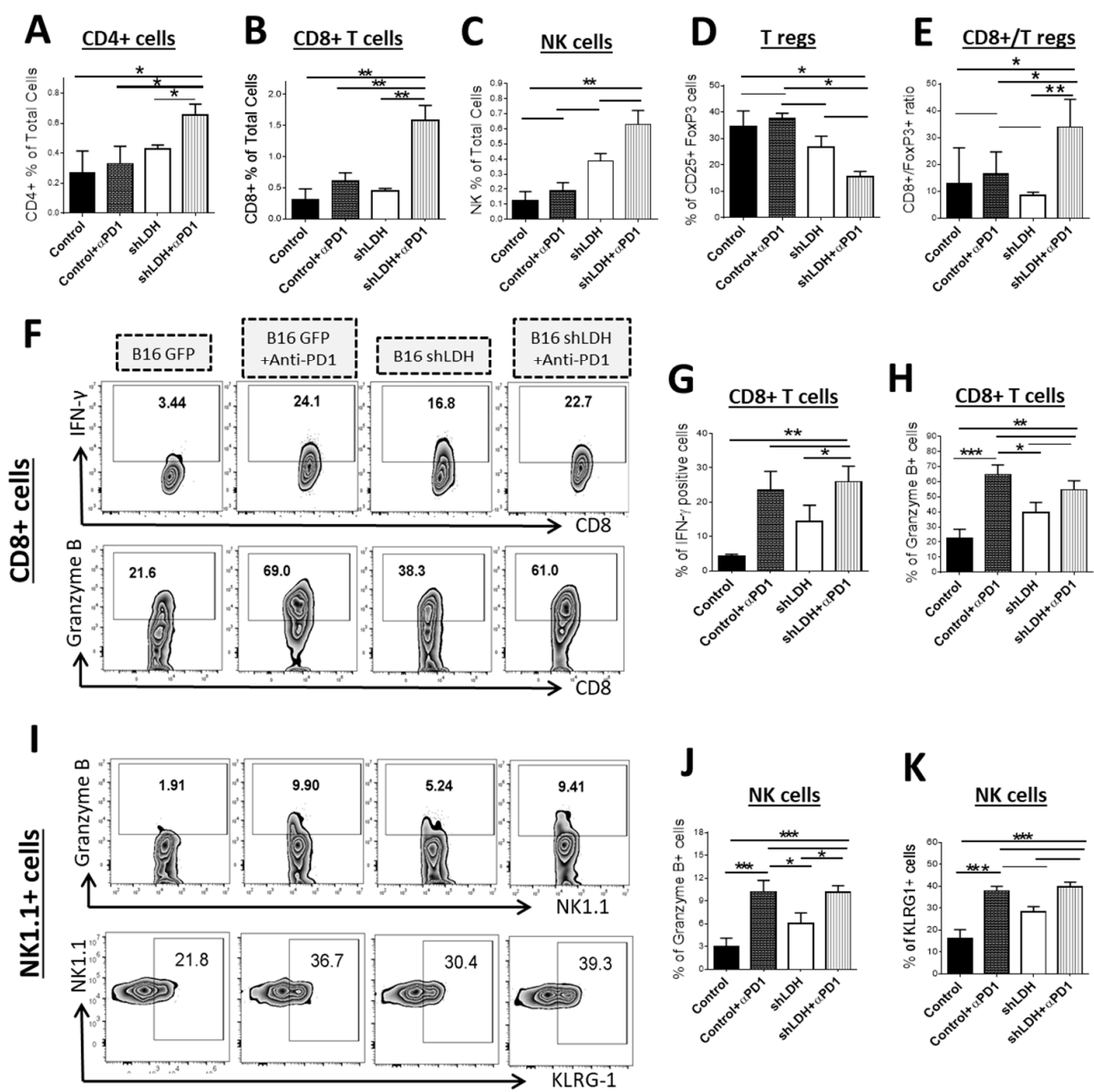

Figure 2. Immunophenotype of intra-tumoral immune cells. (A-D) Intra-tumoral infiltration of CD4 ${ }^{+}$ T-cells (A), CD8 ${ }^{+}$T cells (B), natural killer (NK) cells $(\mathbf{C})$, T regulatory (Treg) cells (D) and CD8 ${ }^{+} /$Treg ratio (E) were evaluated by flow cytometry. The combination of anti-PD-1 use with the deletion of LDH-A in tumor cells induced high infiltration of these cytotoxic cells to the tumor microenvironment. ${ }^{*} p<0.05,{ }^{* *} p<0.01$. (F-H) Levels of granzyme B expression and IFN- $\gamma$ production by intra-tumoral $\mathrm{CD}^{+} \mathrm{T}$ cells were determined using flow cytometry. Either application of anti-PD-1 or deletion of LDH-A in the tumor cells is correlated with higher levels of granzyme B or IFN- $\gamma$ production by infiltrating $\mathrm{CD}^{+} \mathrm{T}$ cells at the tumor site. ${ }^{*} p<0.05$, ${ }^{* *} p<0.01$, ${ }^{* * *} p<0.001$. (I-K). Intra-tumoral infiltrating NK cells also express higher levels of granzyme B in the presence of anti-PD-1 or deletion of LDH-A in the tumor microenvironment. Intra-tumoral NK cells show elevated levels of KLRG-1 on their surface compared to control subjects. ${ }^{*} p<0.05,{ }^{* * *} p<0.001$.

Effector T cells express IFN- $\gamma$ and mediate cytolytic function through the key cytolytic mediators: perforin and granzyme B [12,13]. After $\mathrm{CD} 8^{+} \mathrm{T}$ cells recognize tumor cells, they release their cytolytic granules containing perforin and granzyme B to induce apoptosis in tumor cells [13]. Previous studies have shown that secretion of IFN- $\gamma$ is an essential part of the mechanism of action of CD $8^{+}$tumor infiltrating lymphocytes (TIL) and blocking of IFN- $\gamma$ production will suppress the anti-tumor activity of $\mathrm{CD}^{+} \mathrm{T}$ cells in the tumor microenvironment [12]. We examined IFN- $\gamma$-producing CD8 ${ }^{+}$cells and $\mathrm{NK} 1.1^{+}$cells infiltrating the tumor and found comparable fractions of IFN- $\gamma$ producing cells in mice treated with the anti-PD-1 antibody regardless of the expression of LDH-A in B16-F10 tumors (Figure 2F, top panels). Importantly, mice bearing shLDH-A B16-F10 tumors had elevated IFN- $\gamma$-producing cell 
fractions even without PD-1 antibody treatment (Figure 2F, top panels). A similar profile was observed in granzyme B-expressing CD8 ${ }^{+}$and NK1.1 ${ }^{+} \mathrm{T}$ cells (Figure 2F, bottom panel, and Figure 2I, top panel).

Previous studies have reported that Killer Cell Lectin-Like Receptor Subfamily G positive (KLRG $\left.{ }^{+}\right)$ NK cells have strong protective function against metastatic tumors and high levels of lactate can reduce KLRG expression on NK cells as a mechanism for tumor cells to evade immune response [14]. We found that treatment with PD-1 blocking antibodies increased the fractions of KLRG- $1^{+} \mathrm{NK} 1.1^{+}$ cells in the TME regardless of the expression of LDH-A in B16-F10 tumors. However, in mice bearing shLDH-A B16-F10 tumors, $\mathrm{KLRG}^{+} \mathrm{NK} 1.1^{+}$cells were elevated even without PD-1 antibody treatment (Figure 2I, bottom panels).

\subsection{Infiltrating $C D 8^{+}$CTLs Have Higher Metabolic Activation Status in an LDH-A Deficient Tumor Microenvironment}

Several studies have suggested that cancer cell-derived lactate modifies metabolism and subsequently the function of infiltrating immune cells [1,6]. We next evaluated the mitochondrial status of infiltrating $\mathrm{CD}^{+}$cells. $\mathrm{CD}^{+}$cells had increased levels of mitochondrial mass within their cytoplasm in LDH-A deficient tumors (Figure 3A,B) and this effect was TME-specific, as CD8 ${ }^{+} \mathrm{T}_{\text {cells }}$ in draining lymph nodes did not show an enhanced number of mitochondria (Figure 3C). Scharping et al. have shown that suppression of mitochondrial mass and activity at the TME is a mechanism of immune evasion and drives intra-tumoral $\mathrm{T}$ cell metabolic insufficiency and dysfunction [15]. Therefore, improved mitochondrial function is beneficial foranti-tumor responses. Along with the increase of $\mathrm{CD}^{+} \mathrm{T}$ cell mitochondrial mass in LDH-A deficient tumors, infiltrating CD8 ${ }^{+} \mathrm{T}$ cells express higher levels of reactive oxygen species (ROS) (Figure 3D,E). The latter effect also is tumor site specific and was not seen in draining lymph nodes (Figure 3F).
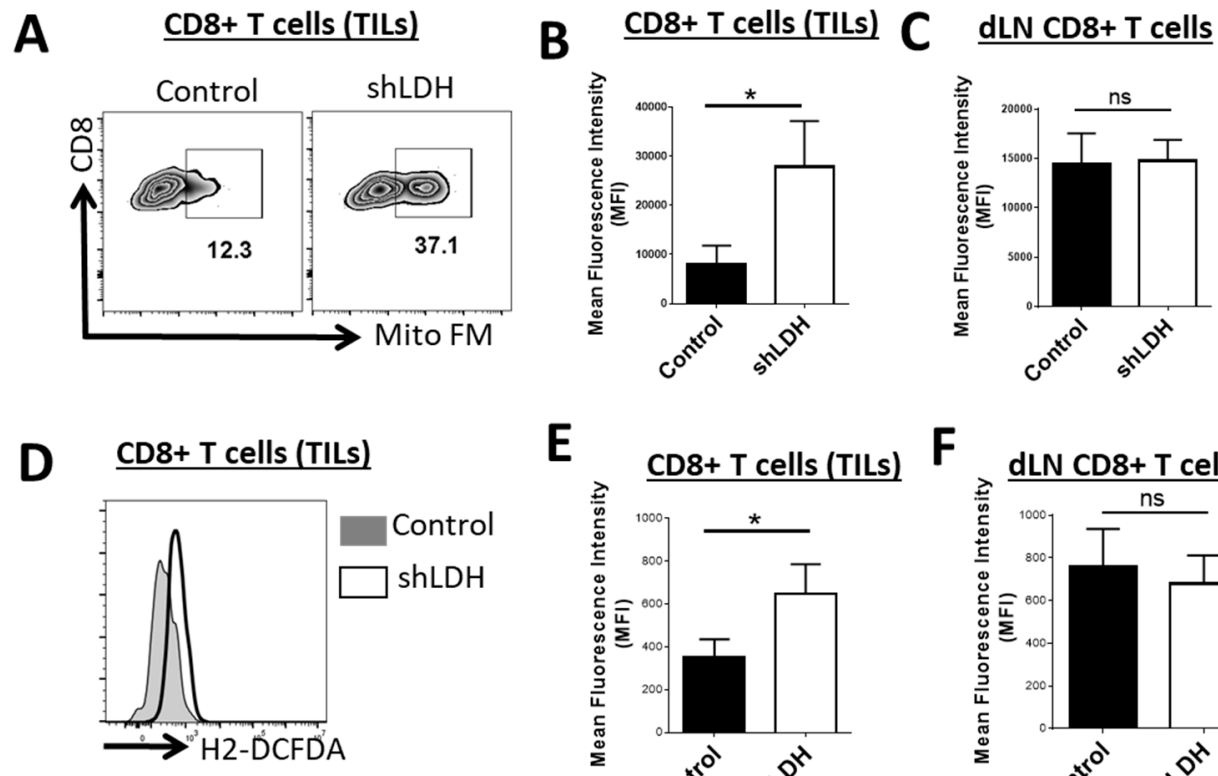

\section{E}

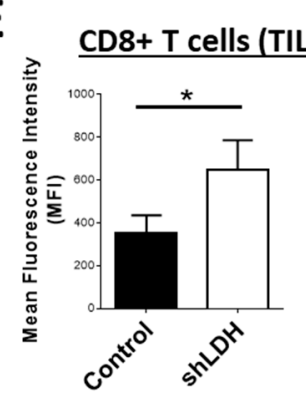

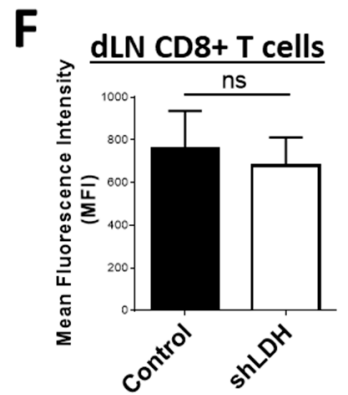

Figure 3. LDH-A deletion in tumor cells is associated with higher levels of mitochondrial mass and intracellular reactive oxygen species (ROS) production in infiltrating CD8 ${ }^{+} \mathrm{T}$ cells. (A-C) Representative flow cytogram of tumor-infiltrating $\mathrm{CD}^{+} \mathrm{T}$ cells (TILs) stained with MitoTracker FM and mean fluorescence intensity (MFI) (bar graph) of the cells from TILs or draining lymph node (dLN). (D-F) TILs or $\mathrm{dLN} \mathrm{CD8}{ }^{+} \mathrm{T}$ cells were stained with the cellular ROS indicator $2^{\prime}, 7^{\prime}$-dichlorodihydrofluorescein diacetate (H2DCFDA). Average MFI (bar graph) demonstrates the higher capacity of CD8 ${ }^{+}$TILs to produce ROS in the LDH-A deficient tumor microenvironment while there is no significant difference in the dLN. ${ }^{*}, p<0.05$. 


\section{4. $C D 8^{+} T$ cells in In Vitro Co-Culture with LDH-A Deficient Tumor Cells Express Higher Levels of Degranulation Markers}

We evaluated $\mathrm{CD}^{+} \mathrm{T}$ cells co-cultured with melanoma cells to assess the specific responses of $\mathrm{T}$ cells in the presence or absence of LDH-A in the tumor cells. CD8 ${ }^{+} \mathrm{T}$ cells harvested from co-culture with shLDH-A melanoma cells demonstrated higher levels of CD107a expression (Figure 4A). CD107a also known as lysosomal-associated membrane protein-1 (LAMP-1) has been described as a substantial marker of $\mathrm{CD}^{+}$T-cell degranulation following stimulation [16]. $\mathrm{CD} 8^{+} \mathrm{T}$ cells cultured with shLDH-A melanoma also had lower expression of CD62L (Figure 4B). In both cases, the addition of anti-PD-1 further enhanced CD107a expression (Figure 4A) and diminished CD62L levels (Figure 4B). Addition of anti-PD-1 to the $\mathrm{CD} 8^{+}$cells: Melanoma co-culture led to higher activity of $\mathrm{CD} 8^{+} \mathrm{T}$ cell mitochondria compared to untreated cells (Figure $4 \mathrm{C}$ ). We also exposed CD8 ${ }^{+} \mathrm{T}$ cells to $10 \mathrm{mM}$ of lactate in vitro. After $24 \mathrm{~h}, \mathrm{CD}^{+} \mathrm{T}$ cells treated with lactate had lower mitochondrial mass (Figure 4D) and lower mitochondrial potential (Figure 4E).

A

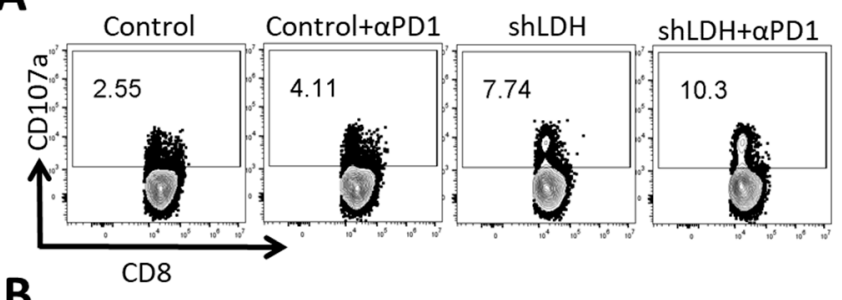

B
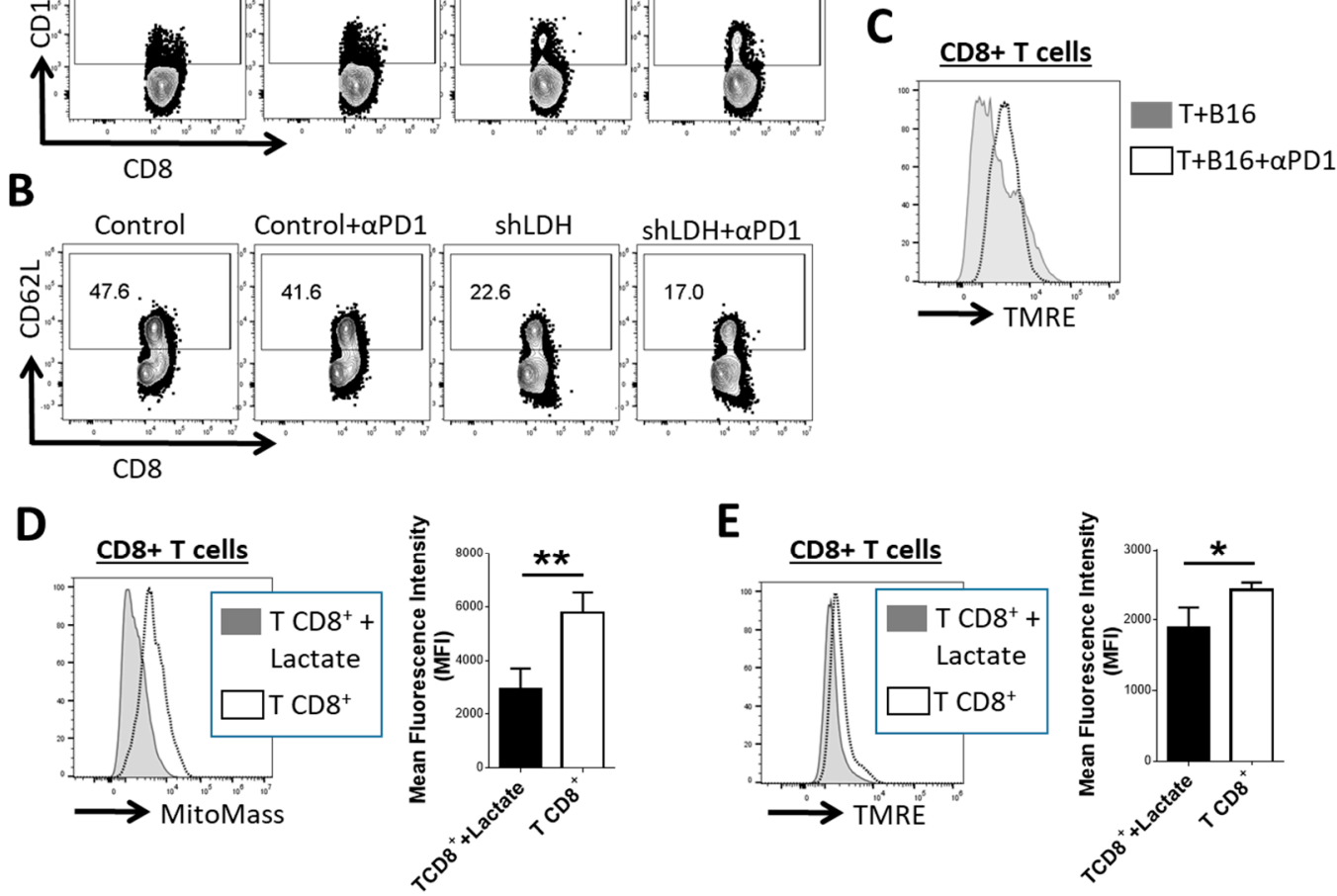

Figure 4. $\mathrm{CD}^{+} \mathrm{T}$ cells co-cultured with LDH-A deletion tumor cells or exposure to lactate in vitro. (A) Representative flow cytogram of a degranulation marker (CD107a) on CD8 ${ }^{+} \mathrm{T}$ cells, co-cultured for $24 \mathrm{~h}$ with LDH-A deleted melanoma cells in vitro. (B) Differential expression of CD62L marker on $\mathrm{CD}^{+} \mathrm{T}$ cells after co-culture for $24 \mathrm{~h}$ in vitro with LDH-A deleted B16-F10 melanoma cells. (C) Effect of added anti-PD-1 on $\mathrm{CD}^{+} \mathrm{T}$ cell's mitochondrial potential (detected by tetra-methylrhodamine ester (TMRE)) while co-cultured with melanoma cells for $24 \mathrm{~h} ; p<0.05$. (D-E) Mitochondrial mass and mitochondrial potential of $\mathrm{CD} 8^{+} \mathrm{T}$ cells exposed to L-lactate $(10 \mathrm{~mm})$ for $24 \mathrm{~h} .{ }^{*} p<0.05 .{ }^{* *} p<0.01$.

\section{Discussion}

The objective of this study was to investigate the role of the metabolic enzyme LDH-A in the modulation of immune anti-tumoral responses to anti-PD-1 immunotherapy. Several studies demonstrated that elevated levels of lactate in the TME due to metabolic switch (Warburg effect) results in the induction of an immunosuppressive milieu that facilitates the growth and invasiveness of tumors by altering the host immune system $[1,5]$. One of the key features of cancer resistance to therapy is 
the induction of co-inhibitory receptors such as checkpoint inhibitors [8]. We showed that melanoma tumor cells augmented the expression of PD-L1 after treatment with lactate, similarly to the lung cancer model [4]. Moreover, blocking of LDH-A in melanoma cells via shRNA prevented the enhancement of PD-L1 expression. Suppression of PD-L1 expression by blockade of LDH-A in melanoma cells was validated in vivo in an LDH-A deficient B16 melanoma tumor model. This enhanced PD-L1 expression would be correlated with metabolic changes in TME.

The rapid growth of tumor cells creates a hypoxic environment, resulting in enhanced glycolytic metabolism and increased lactate in the TME. Lack of LDH-A in myeloid cells resulted in lower hypoxia-inducible factor 1-alpha (HIF1 $\alpha$ ) expression, suggesting a potential mechanism of PD-L1 regulation in response to the LDH-A/lactate axis [4]. HIF1 $\alpha$ has shown to be a target of lactate in cancer cells [17]. Other studies have also shown that hypoxia mediated through HIF1 $\alpha$ regulates expression of PD-L1 [18]. Moreover, studies using human lung cancer models suggested alteration of PD-L1 by lactate through G protein-coupled receptor 81(GPR81) in a TAZ-dependent pathway [19]. Recently, another group highlighted pyruvate, an upstream metabolite of lactate, as a regulator of PD-L1 expression via induction of the bone morphogenetic protein 4/phosphorylated SMAD1/5/IFN regulatory factor 1(1BMP4/p-SMAD1/5/IRF1) signaling pathways [20].

Our results show that LDH-A-deficient tumors are significantly more responsive to anti-PD-1 treatment compared to control tumors expressing LDH-A. These results are in line with previous studies on advanced/metastatic melanoma patients treated with anti-PD-1. This study demonstrated that patients with an elevated baseline LDH-A had significantly shorter survival and elevation of serum LDH-A correlated with progressive disease [21]. Blocking of LDH-A in tumor cells effectively enhances infiltration of $\mathrm{CD} 8^{+} \mathrm{T}$ cells and NK cytotoxic cells in the TME. These infiltrating NK cells and $\mathrm{CD}^{+}$cytolytic cells expressed higher amounts of granzyme B in the TME of tumors in mice treated with anti-PD-1 therapy. Moreover, intra-tumoral NK cells had elevated levels of KLRG-1 on their surface. KLRG-1 is a marker of terminally differentiated NK cells and T cells that binds to several members of the cadherin family. KLRG-1 is considered as an inhibitory receptor. However, the anti-tumoral function of KLRG-1 ${ }^{+}$NK cells in the TME was indicated in pulmonary metastatic disease [14]. Increased expression of PD-1 on NK cells prevents NK cell-mediated anti-tumor function and is correlated with poor prognosis in digestive cancers [22]. It has been well documented that lactate in the TME induces immunosuppression, which results in T cell and NK cell apoptosis and reduces the frequency of infiltrates into these tumors [23].

In this study, we have examined metabolic alterations of infiltrating $\mathrm{CD} 8^{+} \mathrm{T}$ cells in our treatment groups. Intra-tumoral $\mathrm{CD} 8^{+} \mathrm{T}$ cells demonstrated higher amounts of mitochondrial mass and ROS production accompanied by an increased capacity of IFN- $\gamma$ production in LDH-A deficient tumors. Previous in vitro studies implied that lactate secreted by tumor cells could alter $\mathrm{CD} 8^{+} \mathrm{T}$ cell function and that these functions can be reversed by incubation in lactic acid-free medium for $24 \mathrm{~h}$ [6]. We also showed lower mitochondrial function in response to lactate and higher mitochondrial mass in $\mathrm{CD} 8^{+}$ $\mathrm{T}$ cells treated with anti-PD-1 in co-culture with melanoma cells in vitro. LDH-A deficient tumors facilitate oxidative phosphorylation by shuttling pyruvate into mitochondrial metabolism, resulting in higher production of ROS [24]. Increased levels of ROS are also directly linked to higher mitochondrial biomass in $\mathrm{CD}^{+} \mathrm{T}$ cells in LDH-A deficient tumors. Prior studies showed the importance of mitochondrial biogenesis during early effector maturation of $\mathrm{CD} 8^{+} \mathrm{T}$ cells [25]. However, abrogation of mitochondria-derived ROS in PD- $1^{+} \mathrm{CD} 8^{+}$TILs by a protein kinase R-like endoplasmic reticulum kinase (PERK) inhibitor bolstered $\mathrm{CD} 8^{+}$TIL viability [26].

Lactic acid from melanoma cells inhibited tumor associated antigen (TAA)-triggered IFN- $\gamma$ secretion in three-dimensional (3D) melanoma spheroid co-cultures [27]. Mendler et al. similarly confirmed that lactic acidosis reduced T cell receptor (TCR)-driven IFN- $\gamma$, tumor necrosis factor-alpha (TNF- $\alpha$ ) and IL-2 expression and CTL functions, suggesting MAPKs p38 and JNK/c-Jun signaling pathways as the mechanism of suppression [28]. Another study reported that lactic acid and acidification 
diminish nuclear factor of activated T-cells (NFAT) levels resulting in diminished IFN- $\gamma$ production and prevent $\mathrm{T}$ cell and NK cell activation [23].

Therefore, blocking of LDH-A and PD-1 might shift the tumor milieu towards pro-inflammatory and anti-tumor responses due to the activation of mitochondria and ROS-mediated signaling. Indeed, increased ROS levels in the TME enhanced IFN- $\gamma$-induced apoptosis of colon cancer cells [29]. However, the levels of ROS implicated in tumor suppression versus promotion are still under debate [30].

Altogether, our results demonstrate higher effectiveness of anti-PD-1 treatment after blockade of LDH-A in melanoma. Such combination treatment strongly blocks the PD-1/PD-L1 pathway, leading to elevation of pro-inflammatory anti-tumor responses such as higher infiltration and activity of NK cells and $\mathrm{CD} 8^{+}$cytotoxic cells and a diminished frequency of Treg cells. Our results indicate that mice with LDH-A deficient tumors treated with anti-PD-1 have low growth due to the additive effects of reduced lactate and decreased tumoral PD-L1 expression which itself is regulated by lactate. Current LDH-A inhibitors are not optimal for in vivo therapies, yet the fast progress of immunometabolic targets and therapies may lead to better strategies to target LDH-A pharmaceutically. Based on our results, we suggest that modulation of LDH-A/lactate could regulate PD-L1 expression and be an effective way to improve the efficacy of anti-PD-1 therapy.

\section{Materials and Methods}

\subsection{Mice}

Female C57BL/6 mice (6-8 weeks old) were purchased from Jackson Laboratories. All animals were held under specific pathogen-free conditions and humanely euthanized by $\mathrm{CO}_{2}$ inhalation according to the standard protocols, which were approved by the Institutional Animal Care and Use Committee (IACUC) at Beth Israel Deaconess Medical Center (Boston, MA) (040-2016, approved 18 August 2018).

\subsection{B16-F10 Melanoma Cell Line and Culture}

B16-F10 melanoma cells (obtained from American Type Culture Collection (ATCC) in 2013) were in stored at passage 3 (used in experiments in up to passage 10), tested for mycoplasma in 2015, and cultured in complete media (CM): Dulbecco's Modified Eagle Medium (DMEM) media supplemented with $10 \%$ heat-inactivated fetal bovine serum (FBS), L-glutamine, $100 \mathrm{mg} / \mathrm{mL}$ streptomycin and $100 \mathrm{U} / \mathrm{mL}$ penicillin. LDH-A was knocked down using shRNA against LDH-A. Scramble shRNA was used as control. To examine the effect of lactate in vitro, B16-F10 melanoma cells $\left(5 \times 10^{5} \mathrm{cell} / \mathrm{mL}\right)$ were cultured in RPMI media with FBS and treated with L-lactate $(1 \mathrm{mmol} / \mathrm{L}$ and $10 \mathrm{mmol} / \mathrm{L})$ or control for $24 \mathrm{~h}$. After incubation with L-lactate, PD-L1 expression was examined by flow cytometry using anti-PD-L1 (clone 10F.9G2, BioLegend, Cambridge, MA, USA). Lactate release from the cultured cells was determined by Lactate Colorimetric Assay Kit II (BioVision Inc., Milpitas, CA, USA).

$\mathrm{CD}^{+} \mathrm{T}$ cells were isolated from the spleen and lymph nodes of C57BL/6 mice using negative selection on magnetic beads (MACS, STEMCELL Technologies, Cambridge, MA, USA) and cultured in presence of $10 \mathrm{mM} \mathrm{L}$-lactate for $24 \mathrm{~h}$ at $37^{\circ} \mathrm{C}$. In another set of experiments, purified CD8 ${ }^{+} \mathrm{T}$ cells were co-cultured with melanoma cells (1:1 ratio) for $24 \mathrm{~h}$ and then degranulation capacity (determined by CD107a staining) and mitochondrial activity were detected.

\subsection{Tumor Model and Anti-PD-1 Treatment}

To establish the tumor model, $2 \times 10^{5}$ of either B16-GFP or B16-shLDH melanoma cells were implanted in to the left flank of C57BL/6 mice. We randomized mice into two experimental arms: (i) treated with anti-PD-1 antibody (clone 29F.1A12, $200 \mu$ g, i.p.) and (ii) control mice injected with of isotype control ( $200 \mu$ g, i.p.) every two days. Anti-PD-1 treatment was applied seven days after cancer cell implantation when the tumors were palpable. Tumor diameters were measured with a digital caliper every two days and tumor volume in $\mathrm{mm}^{3}$ was calculated by the formula: volume $=(\text { width })^{2} \times$ length $/ 2$. 


\subsection{Flow Cytometry}

After harvesting and washing with $1 \times$ PBS, cells were stained with PerCP anti-CD3 (clone 145-2C11, BioLegend, Cambridge, MA, USA), PE anti-mouse CD4 (clone 4B12, Biolegend), FITC anti-CD8 (clone 53-6.7, BioLegend), APC anti-PD-L1 (clone 10F.9G2, BioLegend), PE/Cy7 anti-CD25 (clone PC61, BioLegend), PE anti-FoxP3 (clone 150D, BioLegend), Pacific Blue anti-NK1.1 (clone PK136, BioLegend), APC anti-Granzyme B (clone QA16A02, BioLegend), APC/Cy7 anti-CD107a (clone 1D4B, BioLegend), APC/Cy7 anti-KLRG-1 (clone 2F1/KLRG-1, BioLegend), APC anti-IFN- $\gamma$ (clone XMG1.2, BioLegend). After staining, cells were analyzed by a CytoFLEX flow cytometer (Beckman Coulter, Indianapolis, IN, USA) and then percentage of gated cells was calculated using FlowJo_V10 software (FlowJo, LLC, Ashland, OR, USA).

\subsection{Mitochondrial Mass, Mitochondrial Poetical and Reactive Oxygen Species Production}

Cells were cultured in flow cytometry (FACS) staining buffer (PBS containing 2\% FBS) and incubated with $0.2 \mu \mathrm{M}$ of MitoTracker Deep Red FM (Invitrogen, Eugene, OR, USA) for 30 min at $37^{\circ} \mathrm{C}$. Mitochondrial potential $(\Delta \Psi \mathrm{m})$ was detected by adding $200 \mathrm{nM}$ tetra-methylrhodamine ester (TMRE) (Thermo Fisher, Eugene, OR, USA). For ROS detection, the cells in FACS buffer were treated with $20 \mu \mathrm{M} \mathrm{2} 2^{\prime}, 7^{\prime}$-dichlorodihydrofluorescein diacetate (H2DCFDA; Invitrogen,) for $30 \mathrm{~min}$ at $37^{\circ} \mathrm{C}$. Cells then were washed with FACS buffer and analyzed by a CytoFLEX flow cytometer (Beckman Coulter, Indianapolis, IN, USA).

\subsection{Immunostaining}

Tumor tissues were isolated and frozen in freezing medium using ice-cold methyl butane. Tissues were cut in $6 \mu \mathrm{m}$ sections using a CryoTome (Fisher Scientific, Loughborough, UK); sections were placed on glass slides, then stored at $-80{ }^{\circ} \mathrm{C}$ or immediately used for staining. Tissue sections were then fixed with $2 \%$ paraformaldehyde (PFA) followed by permeabilization with $0.5 \%$ Triton X-100. After blocking with $7 \%$ horse serum (Vector Laboratories, Burlingame, CA, USA) in PBS, primary antibodies were then applied overnight at $4{ }^{\circ} \mathrm{C}$. Sections were then incubated with fluorescently labeled secondary antibodies for $1 \mathrm{~h}$ at room temperature. The images were captured using a Fluorescence Microscope (Zeiss, Thornwood, NY, USA). The following antibodies were used: anti-CD8 (BioLegend), anti-PD-L1 (BioLegend), anti-Phospho-Histone H3 (P-HH3) (Cell Signaling) and anti-CD31 (BD Biosciences).

\subsection{Western Blotting}

For Western blotting, the cells were lysed on ice in modified Radioimmunoprecipitation assay (RIPA) buffer (50 mmol/L TrisHCl (pH 7.4), 50 mmol/L sodium fluoride, $150 \mathrm{mmol} / \mathrm{L} \mathrm{NaCl}, 1 \%$ Nonident P40, 0.5 mol/L EDTA ( $\mathrm{pH}$ 8.0)) supplemented with Complete Proteinase Inhibitor Cocktail (Roche, Mannheim, Germany). Supernatants of the samples were collected after centrifugation at $14,000 \times g$ and protein concentration was determined using a Bicinchoninic Acid Protein Assay Kit (ThermoFisher, Rockfold, IL, USA). Protein (20 $\mathrm{g}$ g of each sample) was electrophoresed on 4-12\% Bis-Tris Gel (Life Technologies, Carlsbad, CA, USA) and transferred to the membrane. The membranes were blocked with 5\% non-fat milk in $1 \times$ Tris-Buffered Saline (TBS) (Boston BioProducts, Ashland, MA, USA) for $1 \mathrm{~h}$ and probed with primary antibodies overnight. Membranes were washed with TBS and visualized using Super Signal West Pico chemiluminescent substrate (ThermoFisher). The following antibodies were used: anti-LDH-A (Abcam, Eugene, OR, USA) and anti- $\beta$-actin (Sigma Aldrich, Burlington, MA, USA).

\subsection{Statistical Analysis}

All data are presented as mean \pm standard deviation (SD) unless otherwise indicated. Comparison between groups was performed using the Student $t$-test or one-way analysis of variance (ANOVA), 
followed by the post-hoc Tukey test. All statistical evaluations were performed using Graph Pad Prism (San Diego, CA, USA) and $p$-values $<0.05$ considered statistically significant.

Supplementary Materials: The following are available online at http://www.mdpi.com/2072-6694/11/4/450/s1, Figure S1: LDH-A Western Blot, Figure S2: Intra-tumor staining, Figure S3: Intra-tumor CD8 ${ }^{+}$infiltration, Figure S4: TC17 frequency.

Author Contributions: Author Contributions: Data curation, S.D and P.S.; Formal analysis S.D., B.W. and P.S; Funding acquisition, B.W. and P.S..; Investigation, S.D., B.W. and P.S.; Supervision, B.W. and P.S.; Writing-original draft, S.D. and P.S.; Writing-review \& editing, S.D., B.W. and P.S.

Funding: Our studies were supported by funding from W81XWH-15-1-0686 to P.S. BW is supported by R01 DK104714.

Conflicts of Interest: The authors declare no conflict of interest. The funders had no role in the design of the study; in the collection, analyses, or interpretation of data; in the writing of the manuscript, or in the decision to publish the results.

\section{References}

1. Romero-Garcia, S.; Moreno-Altamirano, M.M.; Prado-Garcia, H.; Sanchez-Garcia, F.J. Lactate contribution to the tumor microenvironment: Mechanisms, effects on immune cells and therapeutic relevance. Front. Immunol. 2016, 7, 52. [CrossRef] [PubMed]

2. Walenta, S.; Wetterling, M.; Lehrke, M.; Schwickert, G.; Sundfor, K.; Rofstad, E.K.; Mueller-Klieser, W. High lactate levels predict likelihood of metastases, tumor recurrence, and restricted patient survival in human cervical cancers. Cancer Res. 2000, 60, 916-921. [PubMed]

3. Girgis, H.; Masui, O.; White, N.M.; Scorilas, A.; Rotondo, F.; Seivwright, A.; Gabril, M.; Filter, E.R.; Girgis, A.H.; Bjarnason, G.A.; et al. Lactate dehydrogenase a is a potential prognostic marker in clear cell renal cell carcinoma. Mol. Cancer 2014, 13, 101. [CrossRef] [PubMed]

4. Seth, P.; Csizmadia, E.; Hedblom, A.; Vuerich, M.; Xie, H.; Li, M.; Longhi, M.S.; Wegiel, B. Deletion of lactate dehydrogenase-a in myeloid cells triggers antitumor immunity. Cancer Res. 2017, 77, 3632-3643. [CrossRef] [PubMed]

5. Scott, K.E.; Cleveland, J.L. Lactate wreaks havoc on tumor-infiltrating T and NK cells. Cell Metab. 2016, 24, 649-650. [CrossRef]

6. Fischer, K.; Hoffmann, P.; Voelkl, S.; Meidenbauer, N.; Ammer, J.; Edinger, M.; Gottfried, E.; Schwarz, S.; Rothe, G.; Hoves, S.; et al. Inhibitory effect of tumor cell-derived lactic acid on human T cells. Blood 2007, 109, 3812-3819. [CrossRef] [PubMed]

7. Pilon-Thomas, S.; Kodumudi, K.N.; El-Kenawi, A.E.; Russell, S.; Weber, A.M.; Luddy, K.; Damaghi, M.; Wojtkowiak, J.W.; Mule, J.J.; Ibrahim-Hashim, A.; et al. Neutralization of tumor acidity improves antitumor responses to immunotherapy. Cancer Res. 2016, 76, 1381-1390. [CrossRef]

8. Alsaab, H.O.; Sau, S.; Alzhrani, R.; Tatiparti, K.; Bhise, K.; Kashaw, S.K.; Iyer, A.K. PD-1 and PD-L1 checkpoint signaling inhibition for cancer immunotherapy: Mechanism, combinations, and clinical outcome. Front. Pharmacol. 2017, 8, 561. [CrossRef]

9. Ohaegbulam, K.C.; Assal, A.; Lazar-Molnar, E.; Yao, Y.; Zang, X. Human cancer immunotherapy with antibodies to the PD-1 and PD-L1 pathway. Trends Mol. Med. 2015, 21, 24-33. [CrossRef]

10. Ribas, A.; Hamid, O.; Daud, A.; Hodi, F.S.; Wolchok, J.D.; Kefford, R.; Joshua, A.M.; Patnaik, A.; Hwu, W.J.; Weber, J.S.; et al. Association of pembrolizumab with tumor response and survival among patients with advanced melanoma. JAMA 2016, 315, 1600-1609. [CrossRef]

11. Ribas, A.; Shin, D.S.; Zaretsky, J.; Frederiksen, J.; Cornish, A.; Avramis, E.; Seja, E.; Kivork, C.; Siebert, J.; Kaplan-Lefko, P.; et al. PD-1 blockade expands intratumoral memory t cells. Cancer Immunol. Res. 2016, 4, 194-203. [CrossRef] [PubMed]

12. Barth, R.J., Jr.; Mule, J.J.; Spiess, P.J.; Rosenberg, S.A. Interferon gamma and tumor necrosis factor have a role in tumor regressions mediated by murine CD8 ${ }^{+}$tumor-infiltrating lymphocytes. J. Exp. Med. 1991, 173, 647-658. [CrossRef] [PubMed]

13. Lieberman, J. The ABCs of granule-mediated cytotoxicity: New weapons in the arsenal. Nat. Rev. Immunol. 2003, 3, 361-370. [CrossRef] [PubMed] 
14. Renner, P.; Rovira, J.; Klein, C.; Schlitt, H.J.; Geissler, E.K.; Kroemer, A. KLRG1+ natural killer cells protect against pulmonary metastatic disease by immunosurveillance. Oncoimmunology 2014, 3, e28328. [CrossRef]

15. Scharping, N.E.; Menk, A.V.; Moreci, R.S.; Whetstone, R.D.; Dadey, R.E.; Watkins, S.C.; Ferris, R.L.; Delgoffe, G.M. The tumor microenvironment represses T cell mitochondrial biogenesis to drive intratumoral $\mathrm{t}$ cell metabolic insufficiency and dysfunction. Immunity 2016, 45, 701-703. [CrossRef]

16. Makedonas, G.; Banerjee, P.P.; Pandey, R.; Hersperger, A.R.; Sanborn, K.B.; Hardy, G.A.; Orange, J.S.; Betts, M.R. Rapid up-regulation and granule-independent transport of perforin to the immunological synapse define a novel mechanism of antigen-specific $C D 8^{+} \mathrm{T}$ cell cytotoxic activity. J. Immunol. 2009, 182, 5560-5569. [CrossRef]

17. Colegio, O.R.; Chu, N.Q.; Szabo, A.L.; Chu, T.; Rhebergen, A.M.; Jairam, V.; Cyrus, N.; Brokowski, C.E.; Eisenbarth, S.C.; Phillips, G.M.; et al. Functional polarization of tumour-associated macrophages by tumour-derived lactic acid. Nature 2014, 513, 559-563. [CrossRef]

18. Noman, M.Z.; Desantis, G.; Janji, B.; Hasmim, M.; Karray, S.; Dessen, P.; Bronte, V.; Chouaib, S. PD-L1 is a novel direct target of HIF-1 $\alpha$, and its blockade under hypoxia enhanced mdsc-mediated $t$ cell activation. J. Exp. Med. 2014, 211, 781-790. [CrossRef]

19. Feng, J.; Yang, H.; Zhang, Y.; Wei, H.; Zhu, Z.; Zhu, B.; Yang, M.; Cao, W.; Wang, L.; Wu, Z. Tumor cell-derived lactate induces taz-dependent upregulation of PD-L1 through GRP81 in human lung cancer cells. Oncogene 2017, 36, 5829-5839. [CrossRef]

20. Watanabe, R.; Shirai, T.; Namkoong, H.; Zhang, H.; Berry, G.J.; Wallis, B.B.; Schaefgen, B.; Harrison, D.G.; Tremmel, J.A.; Giacomini, J.C.; et al. Pyruvate controls the checkpoint inhibitor PD-L1 and suppresses T cell immunity. J. Clin. Investig. 2017, 127, 2725-2738. [CrossRef]

21. Diem, S.; Kasenda, B.; Spain, L.; Martin-Liberal, J.; Marconcini, R.; Gore, M.; Larkin, J. Serum lactate dehydrogenase as an early marker for outcome in patients treated with anti-PD-1 therapy in metastatic melanoma. Br. J. Cancer 2016, 114, 256-261. [CrossRef]

22. Liu, Y.; Cheng, Y.; Xu, Y.; Wang, Z.; Du, X.; Li, C.; Peng, J.; Gao, L.; Liang, X.; Ma, C. Increased expression of programmed cell death protein 1 on NK cells inhibits NK-cell-mediated anti-tumor function and indicates poor prognosis in digestive cancers. Oncogene 2017, 36, 6143-6153. [CrossRef]

23. Brand, A.; Singer, K.; Koehl, G.E.; Kolitzus, M.; Schoenhammer, G.; Thiel, A.; Matos, C.; Bruss, C.; Klobuch, S.; Peter, K.; et al. LDHA-associated lactic acid production blunts tumor immunosurveillance by $\mathrm{t}$ and NK cells. Cell Metab. 2016, 24, 657-671. [CrossRef]

24. Xie, H.; Hanai, J.; Ren, J.G.; Kats, L.; Burgess, K.; Bhargava, P.; Signoretti, S.; Billiard, J.; Duffy, K.J.; Grant, A.; et al. Targeting lactate dehydrogenase-A inhibits tumorigenesis and tumor progression in mouse models of lung cancer and impacts tumor-initiating cells. Cell Metab. 2014, 19, 795-809. [CrossRef]

25. Fischer, M.; Bantug, G.R.; Dimeloe, S.; Gubser, P.M.; Burgener, A.V.; Grahlert, J.; Balmer, M.L.; Develioglu, L.; Steiner, R.; Unterstab, G.; et al. Early effector maturation of naive human CD8 ${ }^{+} \mathrm{T}$ cells requires mitochondrial biogenesis. Eur. J. Immunol. 2018, 48, 1632-1643. [CrossRef]

26. Hurst, K.; Lawrence, K.A.; Essman, M.T.; Walton, Z.J.; Leddy, L.R.; Thaxton, J.E. Endoplasmic reticulum stress contributes to mitochondrial exhaustion of CD8 T cells. Cancer Immunol. Res. 2019. [CrossRef]

27. Feder-Mengus, C.; Ghosh, S.; Weber, W.P.; Wyler, S.; Zajac, P.; Terracciano, L.; Oertli, D.; Heberer, M.; Martin, I.; Spagnoli, G.C.; et al. Multiple mechanisms underlie defective recognition of melanoma cells cultured in three-dimensional architectures by antigen-specific cytotoxic t lymphocytes. Br. J. Cancer 2007, 96, 1072-1082. [CrossRef]

28. Mendler, A.N.; Hu, B.; Prinz, P.U.; Kreutz, M.; Gottfried, E.; Noessner, E. Tumor lactic acidosis suppresses ctl function by inhibition of p38 and JNK/c-Jun activation. Int. J. Cancer 2012, 131, 633-640. [CrossRef]

29. Tai, Y.; Cao, F.; Li, M.; Li, P.; Xu, T.; Wang, X.; Yu, Y.; Gu, B.; Yu, X.; Cai, X.; et al. Enhanced mitochondrial pyruvate transport elicits a robust ros production to sensitize the antitumor efficacy of interferon- $\gamma$ in colon cancer. Redox Biol. 2019, 20, 451-457. [CrossRef]

30. Chen, Y.; Zhang, H.; Zhou, H.J.; Ji, W.; Min, W. Mitochondrial redox signaling and tumor progression. Cancers 2016, 8, 40. [CrossRef]

(C) 2019 by the authors. Licensee MDPI, Basel, Switzerland. This article is an open access article distributed under the terms and conditions of the Creative Commons Attribution (CC BY) license (http://creativecommons.org/licenses/by/4.0/). 